\title{
Patient state of knowledge on biosimilars - do physicians need to improve education skills?
}

\author{
Claudia Cobilinschi ${ }^{1,2}$, Daniela Opris-Belinski ${ }^{1,2}$, Catalin Codreanu ${ }^{2,3}$, \\ Razvan Ionescu ${ }^{2,4}$, Magda Parvu ${ }^{4}$, Claudia Mihailov ${ }^{5}$, Horatiu Popoviciu ${ }^{6}$, Codrina Ancuta ${ }^{7}$, \\ Elena Rezus ${ }^{7}$, Ioana Saulescu ${ }^{1,2}$, Sanziana Daia-Iliescul, ${ }^{1,2}$, \\ Laura Groseanu ${ }^{1,2}$, Ruxandra Ionescu ${ }^{1,2}$ \\ ${ }^{1 " S f}$. Maria" Clinical Hospital, Department of Rheumatology and Internal Medicine, \\ Bucharest, Romania \\ 2"Carol Davila" University of Medicine and Pharmacy, Bucharest, Romania \\ 3"Dr. Ion Stoia" Center of Rheumatic Diseases, Bucharest, Romania \\ 4"Colentina" Clinical Hospital, Department of Internal Medicine, Bucharest, Romania \\ 5"Ovidius" University of Medicine, Constanta, Romania \\ ${ }^{6}$ Clinical Emergency Hospital, Tg. Mures, Romania \\ 7"Gr.T. Popa" University of Medicine and Pharmacy, lasi, Romania
}

\begin{abstract}
Availability of biosimilar medicines is rapidly increasing, potentially leading to a wider prescription and a support for healthcare system costs. Despite efforts of patient and physician educational initiatives there still remains a reluctance in prescribing or administering this type of products. A multi-centric survey was conducted on 336 patients with rheumatoid arthritis (RA), spondyloarthritis (SpA), psoriatic arthritis (PsA) or lupus (SLE) on biologic treatment. Half of the patients stated never hearing of biosimilars and one third feared adverse events (infections, cancer). Another $20 \%$ felt insecure on drug tolerability or efficacy. However, the majority declared relying on their rheumatologist if prescribing a biosimilar. Lack of patient information should be accurately addressed by health organizations so that the choice of treatment is done knowingly.
\end{abstract}

Keywords: biosimilars, awareness, patient knowledge, information initiative, nocebo

\section{INTRODUCTION}

The use of biogical therapies has higly impacted the outcome of rheumatic diseases. Biosimilars are close copies of original biological products (1). These products are alternatives to already licensed therapies, which are proved to have clinical efficacy, immunogenicity and good safety profiles (2).

Worldwide prescription of biosimilars has suffered an initial setback because of the lack of understanding in their manufacturing and approval process together with mistrust in the extrapolation of indication in clinical use (3). Lack of biosimilar awareness throughout rheumatic patients results in an unjustified underuse of these drugs followed by deficiencies in economic policies (4). The objective was to assess patients' current knowledge and con- cerns on biosimilars and to investigate their expectations when receiving such a treatment.

\section{MATERIAL AND METHODS}

\section{Study protocol}

We conducted a cross-sectional, multi-centric study using a twelve-item questionnaire that was distributed to Romanian patients with rheumatoid arthritis (RA), spondyloarthritis (SpA), psoriatic arthritis (PsA) or systemic lupus erythematosus (SLE) who were currently on a bio-originator or biosimilar.

\section{Patient population}

336 patients were included in the study and responded to the enquiry during their follow-up visits. 
The survey addressed topics related to biosimilar efficacy, price, accessibility and trust in treating physician. Data was collected from August to December 2018.

Demographic data and treatment history were collected by the same physician. The study was approved by the hospitals' Ethics Committee and all patients gave their written informed consent to complete the questionnaire

\section{Statistical analysis}

The statistical analysis was performed with the aid of SPSS software, version 20.0, setting a significant $P$ value at 0.05 . Data was revealed as mean value \pm standard deviation (SD). Differences between groups were recorded with the aid of Student t-test, whereas Spearman and Pearson tests were used for correlations.

\section{RESULTS}

Out of 336 patients, almost half (47.3\%) had RA, $39.8 \%$ SpA, $12.5 \%$ PsA and only one patient with SLE under belimumab. Patient mean age was 52.5 years.

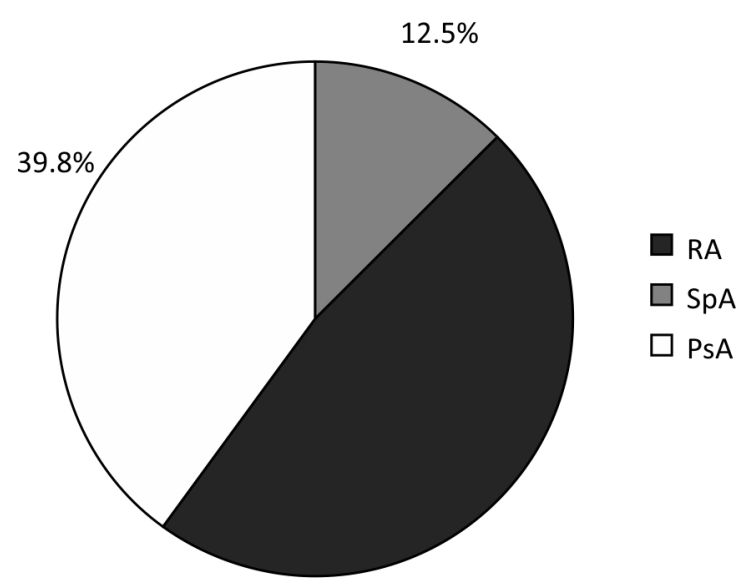

$47.3 \%$

FIGURE 1. Distribution (\%) of patients' rheumatic diseases in the study group

In the study cohort, $13 \%$ were treated with approved biosimilars while $87 \%$ received bio-originators with different mechanisms of action.

A yes/no type of question divided patients into those aware or not of biosimilars, with further exclusion of those with lack of any information. Half of the patients $(48.8 \%)$ stated they had never heard of biosimilars. Surprisingly, four of them were already on this type of treatment. Out of the 172 remaining patients, $28.4 \%$ feared the risk of adverse events like infections or cancer, while almost $20 \%$ expressed either insecurity on drug tolerability or the possibility that the biosimilar might be less efficient that the original drug. Another $19.7 \%$ certified they had no concerns related to these products and only $15.1 \%$ stated confusion regarding the potentially difference in the pharmacological structure of the drugs.

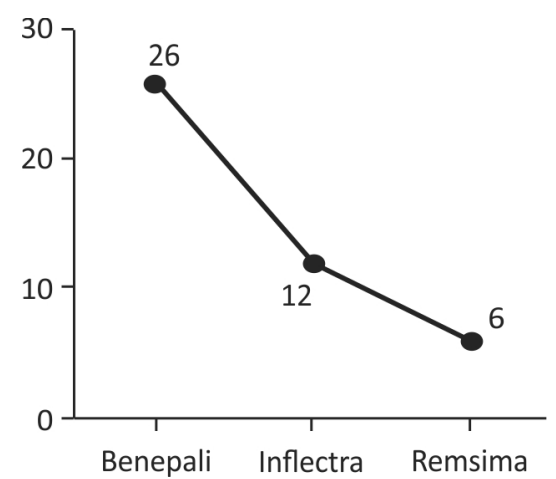

FIGURE 2. Distribution of patients on biosimilars

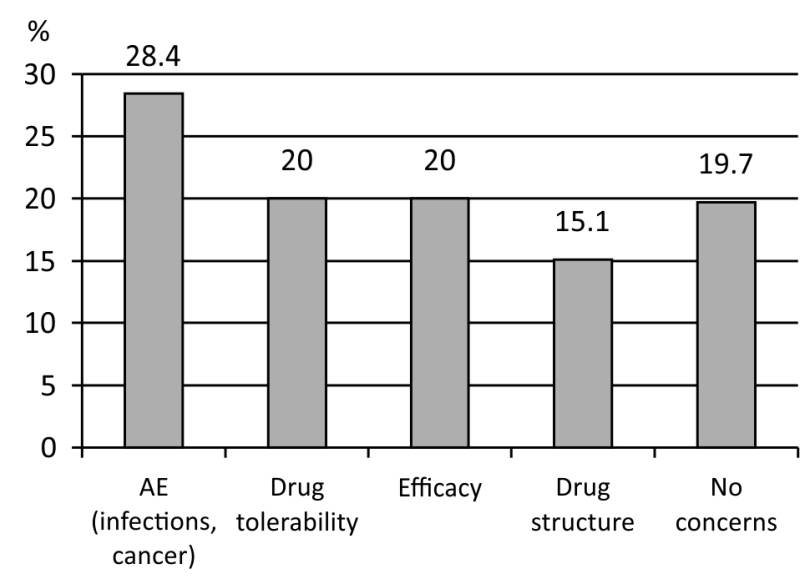

FIGURE 3. Patients' primary concerns on biosimilars

Most patients (48.2\%) are convinced that the price of a drug should not exceed its efficacy or safety, while almost $30 \%$ state that a better price means more patients can have access to treatment. Another $12 \%$ think the price is advantageous for the healthcare while other $11 \%$ think the prince makes no difference.

A striking $52 \%$ of patients are interested in receiving regular updates on drugs, almost $20 \%$ each state that patient associations should express an opinion or that the biosimilar should be tested on more patients before being largely approved. Only $7 \%$ care where the product was conceived or tested.

Half of the respondents say they could accept a switch from an original to a biosimilar if they rheumatologist advises them so and around 30\% might agree but only after being informed. $8.7 \%$ are inter- 
ested in scientific proof of the drug and only $1 \%$ would consent to a change directly from the pharmacist.

If the prescription would only state the biologic, $37.7 \%$ of patients would want to know if it is an original drug or a biosimilar, while $20 \%$ do not mind if they receive either. Another $30 \%$ trust their rheumatologist and $12.7 \%$ would feel more secure if receiving a patient card and written information.

Regarding the change that biosimilars might bring in managing rheumatic diseases, $41 \%$ say it might be a probable change, $12 \%$ feel it is definitely going to change and $26 \%$ do not have any opinion.

Most patients (73.2\%) say that they feel completely confident in their rheumatologist if they would want to prescribe a biosimilar, $18.6 \%$ will have doubts but they will accept the drug and $4 \%$ would ask for another medical opinion.

If the pharmacist would change the physician's initial prescription, $58 \%$ of patients would struggle to obtain the bio-originator, $10 \%$ would accept the change and $7.5 \%$ would embrace it because of the lower price.

After initiation of a biosimilar, $45.9 \%$ would be cautious when administrating it, $23.2 \%$ would stop the drug if an adverse event occurs and $15 \%$ would have no fears during treatment.

\section{DISCUSSIONS}

The results of this study confirm that there is a striking need for additional information of patients on both biologics and biosimilars. The general awareness of rheumatic patients is better regarding biologics than biosimilars, thus indicating a limitation in accepting this type of treatment. Patients' perspective is influenced by the potential side effects, tolerability, the lack of efficacy or the drug structure. Jacobs et al noted the same concerns in respondents but a wider confidence in price and access to biosimilar therapies (5).

A significantly proportion of patients did not mind price issues, although the introduction of biosimilars may reduce the impact of biologics on healthcare costs, allowing an enlargement of treatment choices for both patients and physicians.

Frequently, patients rely solely on their physician to choose the best treatment option despite brief education on the product (6). Apart from medical spe- cialists, both general practitioners and pharmacists should be able to inform target population on biosimilars, their expected results and risks.

However, published studies have shown that not only patients are fully unaware of biosimilar outcomes but also prescribing physicians (7). Despite product official regulations, physicians still point out concerns like benefits on extrapolated indications, traceability, quality, safety profile, efficacy profile and immunogenicity (8).

Once both physicians' and patients' education will expand, there will be an increase not only in initiating treatment with a biosimilar, but also in switching medication from a reference product to a biosimilar (9). In the present study, half of the respondents would be confident to switch on a biosimilar if their rheumatologist advises them so. However, in reluctant patients, the so-called "nocebo" effect can compromise real drug benefits, leading to higher discontinuation rates as Edwards et al. stated (10). The nocebo effect is the negative equivalent of placebo, implying poor disease outcomes in patients that have negative attitudes towards the treatment or low expectations from initiation.

Patients in the study cohort would not agree with the automatic switch done by the pharmacist, namely substitution because there is uncertainty linked to reactions to multiple switching. Pharmacist attitudes may be sensitive to deal with since they are responsible for the implementation of substitution policies at pharmacy level. Previous studies showed that pharmacists may have reservations about assuming such great responsibilities (11).

\section{CONCLUSIONS}

This survey is the first multi-centric study to assess patients' knowledge on biosimilars in Romania. Its results confirm there is still a significant information gap concerning biosimilars in patient population. The majority of them confide the treatment responsibility to their rheumatologist, most concerns coming from adverse event occurrence. Apparently, price matters are not essential criteria for patients. In order to surpass this gap, a more thorough knowledge transfer needs to be made between pharmaceutical companies, physicians, patients and national agencies. 


\section{REFERENCES}

1. Declerck P, Danesi R, Petersel D, Jacobs I. The Language of Biosimilars: Clarification, Definitions, and Regulatory Aspects. Drugs. 2017;77(6):671-77.

2. Kumar R, Sigala S. Biosimilars: Regulatory Status and Implications across the World. J Pharmacovigil. 2016;04(s3).

3. O'Callaghan J, Barry S, Moore U et al. Regulatory Science Ireland: Bridging the information gap on biosimilar medicines. $\mathrm{GaBI} \mathrm{J}$. 2016;5(4).

4. Murphy $P$ et al. Impact of education programme on biosimilar attitudes and beliefs. J Oncol Pharm Pract. 2017;23(8):59-60.

5. Jacobs I, Singh E, Sewell KL, Al-Sabbagh A, Shane LG. Patient attitudes and understanding about biosimilars: An international cross-sectional survey. Patient Prefer Adherence. 2016;10:937-948.

6. O'Callaghan J, Bermingham M, Leonard M et al. Assessing awareness and attitudes of healthcare professionals on the use of biosimilar medicines: A survey of physicians and pharmacists in Ireland. Regul Toxicol Pharmacol. 2017;88:252-261.
7. Schimizzi GF. Biosimilars from a practicing rheumatologist perspective: An overview. Autoimmun Rev. 2016;15(9):911-16.

8. Weise M, Kurki P, Wolff-Holz E, Bielsky MC, Schneider CK. Biosimilars: The Science of Extrapolation. Blood. 2014;124(22):3191-6.

9. Vakil N, Fanikos J. Regulatory and Clinical Perspective on Biosimilars: A Comparison of the US and European Experiences. Curr Emerg Hosp Med Rep. 2019;7(3):111-17.

10. Edwards CJ, Hercogová J, Albrand H, Amiot A. Switching to biosimilars: Current perspectives in immune-mediated inflammatory diseases. Expert Opin Biol Ther. 2019;19(10):1001-14.

11. Beck M, Michel B, Rybarczyk-Vigouret MC et al. Knowledge, behaviors and practices of community and hospital pharmacists towards biosimilar medicines: Results of a French web-based survey. MAbs. 2017;9(2):383-90. 